\title{
Fórmulas hebreas y griegas para expresar los sincronismos en los libros de 1 y 2 Reyes*
}

\author{
David Villar Vegas** \\ Universidad Complutense de Madrid \\ ORCID ID: https://orcid.org/0000-0002-1106-1154
}

En el Texto Masorético de 1 y 2 Reyes hay una fórmula para expresar los sincronis-

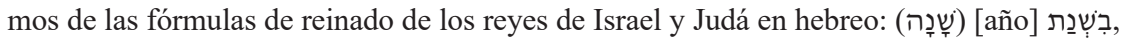

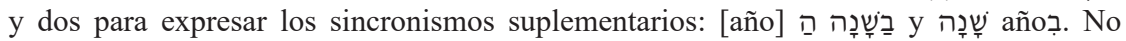
obstante, estas tres expresiones están representadas por cuatro expresiones en griego

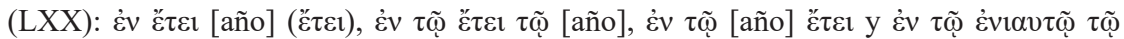
[año]. Nuestro propósito es triple: primero, averiguar por qué hay tres fórmulas diferentes en hebreo para expresar los sincronismos; segundo, explicar por qué sólo una se usa en las fórmulas de reinado y, finalmente, determinar la relación existente entre las fórmulas hebreas y griegas para comprobar si la Fuente Hebrea del Antiguo Griego (OG) fue o no diferente.

Palabras Clave: Reyes de Israel y Judá; Texto Masorético; Septuaginta (LXX); fórmulas de reinado; Antiguo Griego (OG).

Hebrew and Greek Formulas for Expressing the Synchronisms in 1-2 Kings.- In the Masoretic Text of the 1-2 Kings there is one formula for expressing the synchronisms

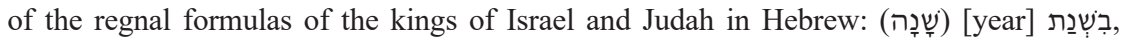

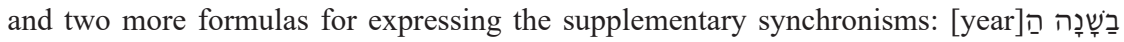
and בow However, these three expressions are represented by four expressions in

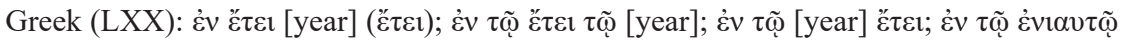
$\tau \tilde{\omega}$ [year]. Our purpose is threefold: firstly, to find out why there are three different formulas in hebrew to express synchronisms; secondly, to explain why only one is used in the regnal formulas; and finally, to determine the relationship between the Hebrew and the Greek formulas to verify whether the Hebrew source of the Old Greek text (OG) was different or not.

* La investigación en este trabajo ha sido realizada en el marco de la Beca de Formación del Profesorado Universitario para el proyecto de tesis doctoral titulado «Cronología de los reinos de Israel y Judá: Estudio comparativo del Texto Masorético y de Septuaginta $\left(\mathrm{LXX}^{\mathrm{BL}}\right) »($ FPU 15/01041), financiada por el actual Ministerio de Ciencia, Innovación y Universidades.

**dvillar@ucm.es

Copyright: (C) 2019 CSIC. Este es un artículo de acceso abierto distribuido bajo los términos de la licencia de uso y distribución Creative Commons Reconocimiento 4.0 Internacional (CC BY 4.0). 
KeYwords: Kings of Israel and Judah; Masoretic Text; Septuagint (LXX); Regnal formulas; Old Greek (OG).

\section{INTRODUCCIÓN}

En los libros de 1 y 2 Reyes aparecen una serie de sincronismos para expresar fechas dentro y fuera de las fórmulas de reinado de acceso y de conclusión de los reyes de Israel y Judá. Por un lado, dentro, la forma de expresar los sincronismos en el texto hebreo masorético es la misma

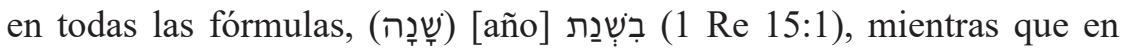
los textos de la Septuaginta aparecen cuatro formas distintas en griego:

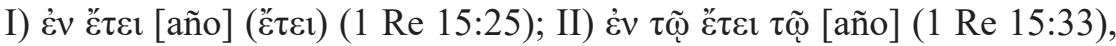
III) $\dot{\varepsilon} v \tau \tilde{\varphi}$ [año] Por otro lado, fuera, aparecen las 4 formas griegas pero, a diferencia del caso anterior, no hay una única forma hebrea $\mathrm{A}$ > בִשְׁנַת [año

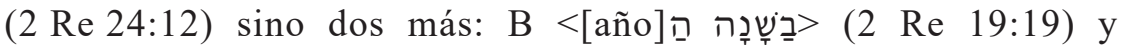
C >

James Donald Shenkel, en su estudio sobre la cronología de los reinos de Israel y Judá, se dio cuenta de que las expresiones griegas II y IV son traducción de la expresión hebrea $\mathrm{B}$ y que esta estaría presente también en la presunta fuente hebrea del texto griego, en al menos dos sincronismos de las fórmulas de reinado: el de Josafat de 1 Re 16:28a y el de Asá de $1 \operatorname{Re~15:91.~}$

Este hecho implica que la forma de expresar los sincronismos de las fórmulas de reinado en el Texto Masorético (en adelante TM) de 1 y 2 Reyes y en el de la supuesta fuente hebrea del Griego Antiguo (en adelante $\mathrm{OG})^{2}$ fue diferente. En el primero únicamente se empleó la forma

1 James D. Shenkel, Chronology and Recensional Development in the Greek Text of Kings (Cambridge: Harvard University Press, 1968) págs. 49-50.

2 Cada vez que hablemos de los textos griegos de la Biblia es muy importante tener clara la diferencia que existe entre LXX y OG. LXX es la abreviatura utilizada para hacer referencia a toda la tradición griega de la Biblia mientras que OG indica la primera traducción hecha desde el hebreo al griego. Esta última se usa a menudo para distinguir ese primer nivel de traducción de formas más tardías del texto griego; James K. AITKEN, The T\&T Clark companion to the Septuagint (New York: Bloomsbury Publishing, 2015) pág. 2. 
A en los sincronismos mientras que en el segundo, al menos, se emplearon la $\mathrm{A}$ y la B.

Sin embargo, el autor no profundiza en esta cuestión y deja los siguientes aspectos sin explicar en lo concerniente a la aparición de formas distintas en hebreo y en griego para expresar los sincronismos de dentro y de fuera de las fórmulas de reinado:

- ¿A qué se debe la aparición de distintas formas en hebreo para expresar los sincronismos? ¿Cuáles son las diferencias entre las expresiones A, B y C?

- ¿Por qué aparecen cuatro formas distintas en griego para traducir los sincronismos de dentro y fuera de las fórmulas de reinado de 1 y 2 Reyes?

- ¿Por qué el TM y el texto de la supuesta fuente hebrea de LXX utilizan, al menos, formas distintas para expresar los sincronismos de las fórmulas de reinado de 1 y 2 Reyes?

El propósito de este trabajo será ofrecer una respuesta a cada una de las tres cuestiones planteadas. Por ello, hemos dividido su estructura en cinco partes: una primera en la que se ubica la presente introducción, tres partes correspondientes a cada uno de los grupos de preguntas, y una última dedicada a las conclusiones. Veámoslas.

2. ¿A QUÉ SE DEBE LA APARICIÓN DE DISTINTAS FORMAS EN HEBREO PARA EXPRESAR LOS SINCRONISMOS? ¿CUÁLES SON LAS DIFERENCIAS

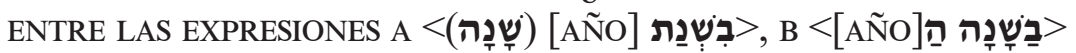

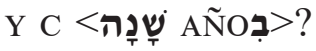

\subsection{Diferencias entre $A, B$ y $C$}

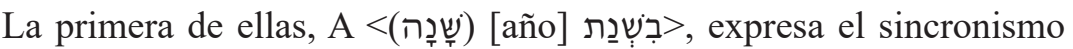
mediante una cadena constructa de dos sustantivos en la que el número va sin el artículo y en forma cardinal pero con significado ordinal ${ }^{3}$. Su

3 Wilhelm Gesenius, Arthur. Cowley y Emil Kautzsch, Gesenius' Hebrew grammar (Oxford: Clarendon Press, 1990), y Paul JoüON, Takamitsu MuraokA y Miguel 
utilización no depende del número expresado en el sincronismo ya que se extiende desde fechas que sincronizan el año primero de un acontecimiento (Ezr 5:13) ${ }^{4}$ hasta fechas que sincronizan el año seiscientos de otro (Gen $7: 11)^{5}$.

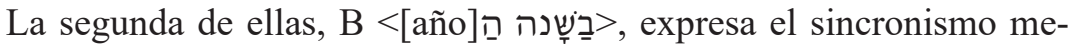
diante un sustantivo, la palabra año, y un adjetivo, el numeral en forma ordinal, ambos determinados. En el TM de la Biblia Hebrea se utiliza para números del 1 al 10, ambos incluidos. Esto se debe a que es una forma en la que se utilizan números ordinales, los cuáles sólo existen para los diez primeros numerales.

En los Óstraca de Samaría, un grupo de textos epigráficos escritos en letra hebrea propia del período monárquico que datan del siglo VIII a. C., aparecen una serie de sincronismos expresados en esta forma B, demostrando su antigüedad ${ }^{6}$. Los sincronismos con números iguales o menores a diez utilizan la escritura hebrea y los mayores se escriben en hierático ${ }^{7}$. Esta forma de escribir los números con signos en hierático está ampliamente atestiguada en diversos territorios de las monarquías de Israel y Judá ${ }^{8}$.

PÉREZ FERnÁNDEZ, Gramática del hebreo bíblico: Instrumentos para el estudio de la Biblia (Navarra: Verbo divino, 2007).

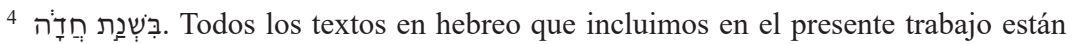
tomados de la siguiente edición de la Biblia: Rudolf Kittel, (ed.), Biblia Hebraica Stuttgartensia (Stuttgart: Deutsche Bibelgesellschaft, 1997).

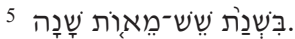

6 John C. L. Gibson, Textbook of Syrian Semitic Inscriptions: Hebrew and Moabite Inscriptions (London: Oxford University Press, 1971) págs. 8-11.

7 William H. SHEA, «The Date and Significance of the Samaria Ostraca», Israel Exploration Journal 27 (1977) págs. 16-27.

8 Yohanan Aharoni, «The Samaria Ostraca - An Additional Note», Israel Exploration Journal 12 (1962) págs. 67-69, y «The Use of Hieratic Numerals in Hebrew Ostraca and the Shekel Weights», Bulletin of the American Schools of Oriental Research (BASOR) 184 (1967) págs. 13-19; Solomon A. Birnbaum, «The Dates of the Gezer Tablet and of the Samaria Ostraca», Palestine Exploration Quarterly 74 (1942) págs. 104108; Meindert Dijkstra, «Chronological Problems of the Eighth Century BCE: A New Proposal for Dating the Samaria Ostraca», en Past, Present, Future: The Deuteronomistic History and the Prophets, eds. Harry F. Van Rooy y Johannes C. De Moor (Leiden: Brill, 2000) págs. 76-87; Ivan Tracy Kaufman, «The Samaria Ostraca: An Early Witness to Hebrew Writing», Biblical Archaeologist 45 (1982) págs. 229-239; David GordoN 
La última de las expresiones hebreas que aparecen en la Biblia para

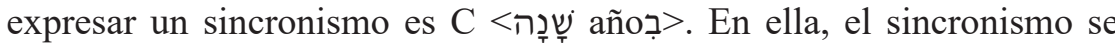
expresa con el numeral en forma cardinal precedido por la preposición ב y seguido por la palabra שָָָָ. Esta forma de expresar el sincronismo, tanto en 1 y 2 Reyes como en el resto de la Biblia Hebrea, únicamente se utiliza con numerales mayores de diez.

Tras explicar las características de cada una de las tres formas de expresar los sincronismos en hebreo, hemos comprobado que la primera se utiliza con todos los numerales independientemente de la cifra que expresen, que la segunda limita su uso del 1 al 10 incluidos -salvo en hebreo del período monárquico, como reflejan los Óstraca de Samaríay que la tercera se usa únicamente con numerales mayores de diez. ¿Por qué entonces aparecen en mismos textos como en Reyes las tres formas? ¿Por qué tenemos en la Biblia sincronismos de cifras iguales expresados de formas distintas?

\section{2. ¿A qué se debe la aparición de distintas formas en hebreo para expresar los sincronismos?}

Si excluimos de nuestro análisis el TM de 1 y 2 Reyes, que son los libros que -junto con los de 1 y 2 Samuel $^{9}$, y tal y como ya han demos-

Lyon, «Hebrew Ostraca from Samaria», Harvard Theological Review (HTR) 4 (1911) págs. 136-143; Benjamin MAZAR, «The Historical Background of the Samaria Ostraca», Journal of the Palestine Society 21 (1948) págs. 117-133; Alan R. Millard, «Strangers from Egypt and Greece - The Signs for Numbers in Early Hebrew», en Immigration and Emigration within the Ancient Near East: Festschrift E. Lipinski, eds. Edward LIPINSKI, Karel VAN LeRBERGHE y Antoon SchoORS (Leuven: Peeters, 1995) págs. 189-194; William H. SHEA, «Israelite Chronology and the Samaria Ostraca», Zeitschrift Des Deutschen Palästina-Vereins 101 (1985) págs. 9-20, y Yigael YADIN, «A Further Note on the Samaria Ostraca», Israel Exploration Journal 12 (1962) págs. 64-66.

${ }^{9}$ Un elemento que goza de gran aceptación dentro de la historia del texto de la Septuaginta (LXX) es que la transmisión textual de los libros de 1 y 2 Samuel y 1 y 2 Reyes fue de la mano; Julio Trebolle, «Qumran Fragments of the Book of Kings», en The Book of Kings: Sources, Composition, Historiography and Reception, eds. André LÉMAIRE y Baruch HALPERn (Leiden: Brill, 2010) págs. 19-40: 19. Ello es porque en LXX los libros de Samuel y de Reyes son un solo libro dividido en cuatro partes, B $\alpha \sigma i \lambda \varepsilon i \tilde{\omega} v$, de los Reyes. Por ello en la Septuaginta 1 y 2 Samuel se conocen como 1 
trado muchos autores ${ }^{10}$ - más diferencias tienen con la supuesta fuente hebrea de debajo del $\mathrm{OG}^{11}$ y no conviene, por tanto, utilizar sus datos, encontramos solución a la pregunta viendo cómo aparecen estas expresiones en el resto de los libros de la Biblia Hebrea, siendo conscientes de que algunos de ellos pueden presentar problemas parecidos a los de 1 y 2 Reyes, como Zacarías y Ageo, aunque no de la misma forma e intensidad.

Existe una división generalmente aceptada dentro del ámbito de estudio de la crítica literaria y textual de la Biblia Hebrea que es la que se basa en el tipo de hebreo que aparece en cada uno de sus libros. Según el hebreo que aparezca, se ha dividido a los libros bíblicos en 3 grupos distintos:1) los representantes del Early Biblical Hebrew; esto es, la lengua de los antiguos poemas de la Biblia Hebrea como Gen 49; 2) los representantes del Classical Biblical Hebrew, presente en la mayoría de los libros de la Biblia Hebrea, que constituye el hebreo del período del Primer Templo, y 3) los representantes del Late

y 2 Reinos y 1 y 2 Reyes como 3 y 4 Reinos. No obstante, en este trabajo utilizamos la denominación más común contenida en el TM por la cual se hace referencia a los libros de Reyes como 1 y 2 Reyes y a los de Samuel como 1 y 2 Samuel; Bill T. Arnold, H. G. M. Williamson y Rubén Gómez, Diccionario del Antiguo Testamento: Históricos (Barcelona: Clie, 2014) pág. 1662.

10 Anneli Aejmelaeus, «A Kingdom at Stake: Reconstructing the Old Greek - Deconstructing the Textus Receptus», en Scripture in Transition: Essays on Septuagint, Hebrew Bible, and Dead Sea Scrolls in Honour of Raija Sollamo, eds. Anssi Voitila y Jutta JoKiranta (Leiden: Brill, 2018) págs. 352-366; Julio Trebolle, «El estudio de 4QSama: implicaciones exegéticas e históricas», Estudios Bíblicos 39 (1981) págs. 5-18 $\mathrm{y}$ «From the Old Latin through the Old Greek to the Old Hebrew (2 Kings 10:23-25)», Textus 11 (1984) págs. 17-36; Andrés Piquer Otero, «What Text to Edit?: The Oxford Hebrew Bible edition of 2 Kings 17,1-23», en After Qurmrán: Old and Modern Editions of the Biblical Texts - The Historical Books, eds. Hans Ausloos, Bénédicte LemmeliJn y Julio Trebolle (Leuven: Uitgeverij Peeters, 2012) págs. 227-243, y Tuukka KaUHANEN, Lucifer of Cagliari and the Text of 1-2 Kings (Atlanta: SBL Press, 2018).

11 Gershon GaLIL, The Chronology of the Kings of Israel and Judah (Leiden: Brill, 1992); Jeremy Hughes, Secrets of the Times: Myth and History in Biblical Chronology (Sheffield: JSOT Press, 1993); M. Christine Tetley, The Reconstructed Chronology of the Divided Kingdom (Winona Lake: Eisenbrauns, 2005), y Julio TrebolLe, «Redaction, Recension, and Midrash in the Books of Kings», en Reconsidering Israel and Judah, ed. Gary N. KNOPPERs (Winona Lake: Eisenbrauns, 2000) págs. 475-492. 
Biblical Hebrew, la lengua de los libros cuya fecha post-exílica es evidente ${ }^{12}$.

Si aplicamos este criterio a en qué libros aparecen cada una de las tres expresiones hebreas salen a la luz las siguientes evidencias:

1. La expresión hebrea A se localiza en libros que se ubican dentro del grupo del Late Biblical Hebrew en 23 de las $28^{13}$ veces que aparece.

2. La expresión hebrea B lo hace en el grupo de libros perteneciente al Classical Biblical Hebrew en 23 de las $28^{14}$ veces que aparece.

3. Por último, la expresión hebrea $\mathrm{C}$ hace lo propio en libros pertenecientes al grupo del Classical Biblical Hebrew en 17 de las $18^{15}$ veces que aparece.

Por lo tanto, tras este análisis, a la doble pregunta de a qué se debe la aparición de distintas formas en hebreo para expresar los sincronismos y cuáles son las diferencias entre las expresiones A, B y C respondemos con lo siguiente:

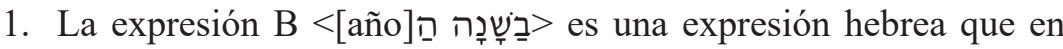
los libros de la Biblia únicamente aparece para expresar un sincronismo en forma ordinal con números del 1 al 10. Se encuentra en los libros del grupo del Classical Biblical Hebrew y también

12 Utilizamos la terminología inglesa para clasificar los distintos tipos de hebreo ya que esta es la que aparece en la obra del autor que trabaja esta cuestión: Steven E. FASSBERG, «What is Late Biblical Hebrew?», Zeitschrift Für Die Alttestamentliche Wissenschaft 128 (2016) págs. 1-15.

13 A saber, en: Dan 1:1, Dan 2:1, Dan 7:1, Dan 8:1, Dan 9:1, Dan 9:2, Dan 10:1, Dan 10:10, Dan 11:1, Gen 7:11, 2 Cr 3:2, 2 Cr 13:1, 2 Cr 16:1, 2 Cr 16:12, 2 Cr 17:7, 2 Cr 34:8, Ezr 1:1, Ezr 5:13, Ezr 6:3, Ezr 7:7, Neh 13:6, Est 1:3, Est 2:6, Est 3:7, Hag 1:1, Hag $1: 15$, Zac $1: 7$ y Zac 7:1.

14 A saber, en: Num 10:11, Jer 32:1, Jer 35:1, Jer 36:1, Jer 45:1, 1 Cr 26:31, 2 Cr 12:12, 2 Cr 27:5, Ez 8:1, Gen 47:18, Lev 19:24, Lev 19:25, Lev 25:4, Lev 25:20, Lev 25:21, Num 9:1, Deut 26:12, 2 Cr 23:1, Ezr 3:8, Jer 25:1, Jer 26:2, Jer 28:59, Jer 43:9, Jer 46:1, Jer 52:4, Ez 20:1, Ez 24:1 y Ez 29:1.

15 A saber, en: Gen 8:13, Gen 14:5, Deut 1:3, 2 Cr 34:3b, 2 Cr 35:19, Jer 25:3, Jer 46:2, Jer 52:31, Ez 1:1, Ez 26:1, Ez 29:17, Ez 30:20, Ez 31:1, Ez 32:1, Ez 32:17, Ez 33:21, Ez 40:1a y Ez 40:1b. 
en escritura hebrea propia del período monárquico en los Óstraca de Samaría del siglo viII a. C. En estos óstraca no sólo aparece con números del 1 al 10, sino también con números mayores escritos en hierático procedentes de Egipto. No podemos saber si esta fue una práctica exclusiva del reino de Israel o también apareció en el reino de Judá. Lo que sí podemos intuir es que esta práctica ilustraba la no existencia de números en forma ordinal mayores que diez. Por ello, parece que se utilizó una expresión complementaria a $\mathrm{B}$, que empleaba números en forma cardinal pero con sentido ordinal para mayores de diez. Tal expresión

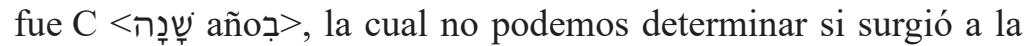
vez que $\mathrm{B}$ o no, ${ }^{16}$ aunque sí parece que coexistieron en el tiempo, ya que en los libros pertenecientes al grupo del Classical Biblical Hebrew, donde ambas aparecen, B se utilizó con números iguales o menores a diez y $\mathrm{C}$ con los mayores.

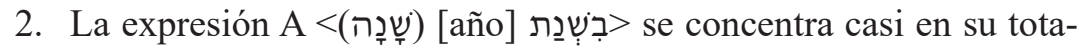
lidad en el grupo de libros del Late Biblical Hebrew. Por ello, es posible que esta expresión apareciera en el período persa tardío del judaísmo, o aun en el helenístico, como deseo de unificar la manera de expresar los sincronismos bajo una única forma. De ahí que casi no se encuentre salvo contadas excepciones en los libros con un hebreo más antiguo y que aparezca en su práctica totalidad en los libros con un hebreo más tardío ${ }^{17}$.

${ }^{16}$ De ello depende la extensión de la práctica de expresar los números $>10$ en sentido ordinal en hierático. Si esta práctica fue extendida y también se aplicó en Judá, puede ser que la expresión $C$ surgiera para no utilizar números en una escritura extranjera y hacerlo todo en un sistema de escritura hebrea con dos expresiones. Si no fue extendida, quizás únicamente la tenemos en el reino del Norte y la expresión $\mathrm{C}$ pudo coexistir con B en Judá en el período del Primer Templo. Apoyamos en este trabajo la primera opción ya que se puede apreciar que en el Pentateuco la expresión B aparece 9 veces, por 3 de la C, indicando quizá esa anterioridad.

17 A este respecto cabe destacar también las diferencias que se dan en el uso de estas fórmulas entre los libros de la Biblia que presentan textos paralelos, como Reyes, Crónicas, Isaías y Jeremías. Todos los textos paralelos, salvos los dos que indicaremos, emplean las mismas expresiones en los sincronismos. Los dos casos distintos son los de

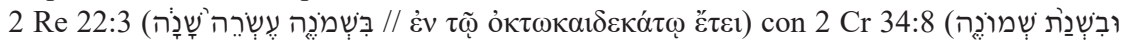

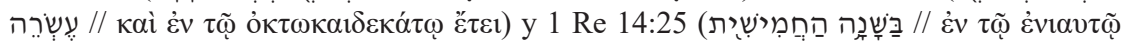

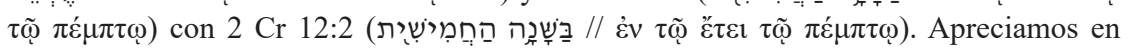


Después de haber resuelto la pregunta sobre la utilización de expresiones distintas para formular los sincronismos en hebreo, surge el siguiente y lógico interrogante: ¿qué repercusión tienen estos resultados en los Libros de 1 y 2 Reyes?

Con estos resultados en la mano ¿por qué la expresión A que aparece en su práctica totalidad en libros que pertenecen al grupo de Late Biblical Hebrew aparece en exclusividad en las fórmulas de reinado de un libro que a priori no pertencería a ese grupo? ¿Ha habido una reescritura de 1 y 2 Reyes? o ¿quizás no hubo tal reescritura y este hecho depende de la versión utilizada?

Shenkel demostró ${ }^{18}$, basándose en los textos griegos considerados como OG, que en al menos dos fórmulas de reinado de 1 Reyes la expresión utilizada por la supuesta Vorlage hebrea de OG fue la B, distinta a la del TM y, con lo que se ha visto ahora, también anterior. ¿Por qué aparece en estos dos casos B debajo del OG y TM tiene la expresión A? ¿Hay más diferencias en el resto de 1 y 2 Reyes? Para resolver esta cuestión debemos contestar la segunda pregunta planteada en la Introducción y averiguar si el uso de distintas expresiones en griego obedece o no al uso de distintas expresiones en hebreo.

\section{3. ¿POR QUÉ APARECEN CUATRO FORMAS DISTINTAS EN GRIEGO PARA TRADUCIR LOS SINCRONISMOS DE DENTRO Y FUERA DE LAS FÓRMULAS DE REINADO DE 1 Y 2 REYES?}

Para resolver estas preguntas veremos a continuación la relación que existe en los Libros de 1 y 2 Reyes entre las expresiones griegas I $<\dot{\varepsilon} v$ हैं $\tau 1$

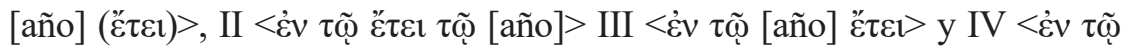

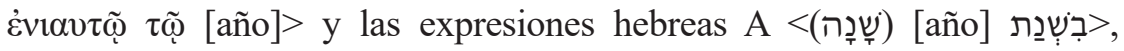

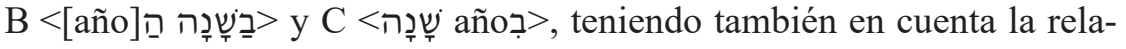

ambos como la fórmula de Crónicas es distinta de la de Reyes, siendo en Reyes en el primer caso la expresión C la utilizada y en el segundo la B mientras que en Crónicas en los dos casos se utiliza la A.

18 SHENKel, CRD, págs. 49-50. 
ción que tengan estas expresiones en el resto de los textos de la Biblia Hebrea.

Es importante que a la hora de determinar la relación entre las expresiones griegas y las hebreas fijemos en cada uno de los textos cuál es la lectura del OG, ya que sabemos que desde el momento en el que estos textos fueron traducidos del hebreo al griego su contenido se fue adaptando al del texto hebreo dominante ${ }^{19}$.

Dicho esto, pasemos a analizar las expresiones griegas II $<\dot{\varepsilon} v \tau \tilde{\omega}$ ह้ $\tau \varepsilon \iota$

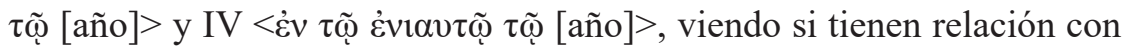

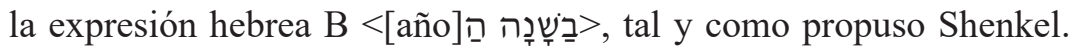

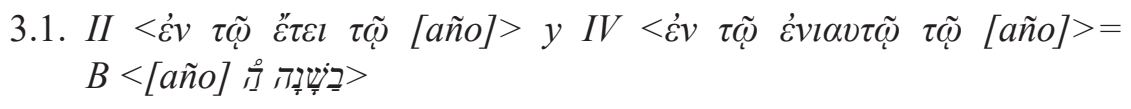

\subsubsection{La expresión griega II}

En primer lugar, hay seis sincronismos en los libros de 1 y 2 Reyes que sabemos que tienen una lectura $\mathrm{OG}^{20}$ y en los que la expresión

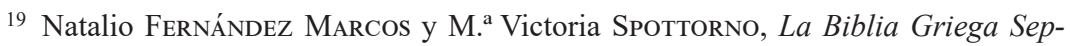
tuaginta (Salamanca: Ed. Sígueme, 2008-2013), y Pablo A. ToriJAno, «How much Hexaplaric Material Entered into the Antiochene Textual Tradition?», en The Legacy of Barthélemy: 50 Years after Les Devanciers d'Aquila, eds. Anneli AejMelaeus y Tuukka KAUHANEN (Göttingen: Vandenhoeck\&Ruprecht, 2017) págs. 69-119.

20 En este trabajo aceptamos la división de Samuel-Reyes en cuatro secciones propuesta por Thackeray (Henry St. John THACKERAY, «The Greek Translators of the Four Books of Kings», Journal of Theological Studies 8 [1907] págs. 262-278) en las que $\beta \gamma$ (2 Samuel $10-1$ Reyes 2) y $\gamma \delta$ (1 Reyes 22 - 2 Reyes 25) son las secciones kaige, tal y como ha sido aceptado desde la publicación de BARThÉLemy, Devanciers. En estas secciones, el Códice Vaticano reproduce el texto de la revisión kaige, mientras que los manuscritos antioquenos preservan un texto que es prácticamente idéntico al del OG; Andrés Piquer, Pablo Torijano y Julio Trebolle «Septuagint Versions, Greek Recensions and Hebrew Editions: The Text-critical Evaluation of the Old Latin, Armenian and Georgian Versions in III-IV Regnorum», en Translating a translation, eds. Hans AusLoos, Johann Cook y Florentino García Martínez (Leuven: Uitgeverij Peeters, 2008) págs. 251-281. La revisión kaige fue un proyecto o tradición de no uniformes revisiones hecha por un grupo de autores en varios siglos, s. I a. C. - I d. C., que tuvo como objetivo adaptar el texto de LXX al del hebreo proto-TM dominante; Leonard J. GreENSPOON, «The Kaige Recension: The Life, Death, and Postmortem Existence of a Modern 


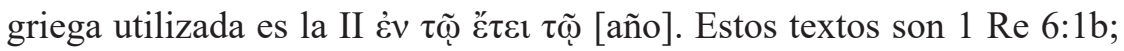
$1 \operatorname{Re} 15: 33 ; 2 \operatorname{Re} 11: 4 ; 2 \operatorname{Re} 18: 9 ; 2 \operatorname{Re} 19: 29 a . b$.

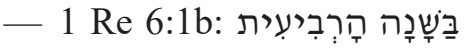

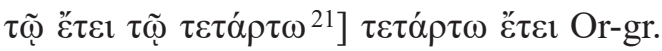

$-1 \operatorname{Re} 15: 33$

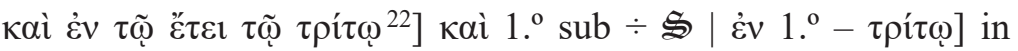

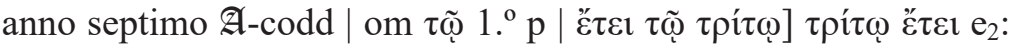
$\pi \varepsilon \mu \pi \tau \omega$ हैं $\varepsilon \iota$ bic $_{2}$ : om $\tau \tilde{\omega} \mathrm{dp}$.

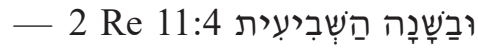

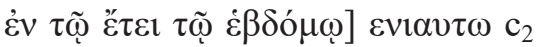

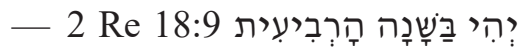

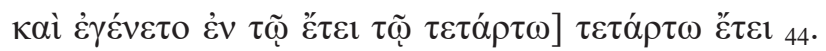

- and Ancient - Phenomenon», en XII Congress of the International Organization for Septuagint and Cognate Studies. Leiden, 2004, ed. Melvin K. H. Peters (Atlanta: Society of Biblical Literature, 2006) págs. 5-16: 5-9. Las otras dos partes de Samuel-Reyes son las llamadas OG o secciones no-kaige $(\alpha=1$ Samuel; $\beta \beta=2$ Samuel 1:1 - 11:1; $\gamma \gamma=1 \operatorname{Re} 2: 12-21: 43)$. En ellas se ha demostrado que el Códice Vaticano es una fuente fiable para la lectura OG (PIQUER, «Minuses», pág. 55), aunque haya casos en los que parece haber sufrido algún tipo de revisión hebraizante también en esas secciones, prefiriéndose entonces el contenido de los manuscritos antioquenos para determinar la lectura OG, aunque también hayan sufrido en esta sección y en la kaige un proceso de revisión; Anneli Aejmelaeus, «Kaige Readings in a Non-Kaige Section in 1 Samuel» en The Legacy of Barthélemy:50 Years after Les Devanciers d'Aquila, eds. Anneli AejmeLAEUS y Tuukka KaUHANEN (Göttingen: Vandenhoeck\&Ruprecht, 2017) págs. 169-184, y Siegfried Kreuzer, «Towards the Old Greek: New Criteria for the Analysis of the Recensions of the Septuagint: Especially the Lucianic Manuscripts and Kaige Recension», en XII Congress of the International Organization for Septuagint and Cognate Studies 2004, ed. Melvin K.H. Peters (Atlanta: SBL, 2006) págs. 239-253.

21 Todos los textos en griego de 1 y 2 Reyes que incluímos en el trabajo se toman de la edición de la Septuaginta de Cambridge: Alan England Brooke y Norman Mclean, (eds.), The Old Testament in Greek: According to the Text of Codex Vaticanus, Supplemented from Other Uncial Manuscripts, with a Critical Apparatus Containing the Variants of the Chief Ancient Authorities for the Text of the Septuagint. Volume 2 (Cambridge-New York-Melbourne: Cambridge University Press, 1930).

$22 \mathrm{Al}$ estar en sección no-kaige, apostamos porque la lectura OG es la mayoritaria y porque los manuscritos antioquenos presentan una lectura particular. 


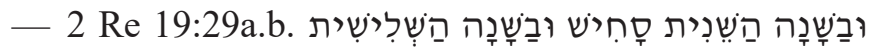

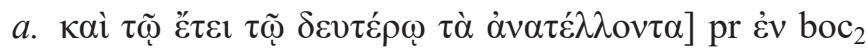

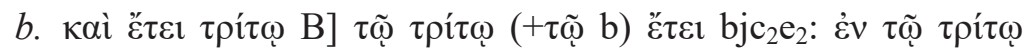

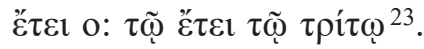

Por tanto, no aceptamos la tesis de Shenkel ${ }^{24}$ de que el traductor de la revisión kaige es el que emplea esta expresión, ya que la misma aparece en los dos primeros textos de la sección OG (1 Re 3-1 Re 21) y es compartida en los de la mencionada sección kaige (1 Re 1-2 y 1 Re 22-2 Re 23) por los manuscritos antioquenos, que apenas se vieron afectados por tal revisión.

En cinco de los seis sincronismos -salvo el de la fórmula de reinado-, la expresión hebrea del TM que aparece es la B, confirmando por tanto la relación apuntada por Shenkel pero matizando que la expresión II no es sólo típica de la sección kaige. Si miramos el resto de libros de la Biblia Hebrea se ve que esta relación también se produce. La expresión griega II aparece 20 veces en los manuscritos de LXX mencionados para determinar el OG (Códice Vaticano y manuscritos antioquenos) y en $18^{25}$ ocasiones la expresión hebrea que tiene detrás es la B. Por tanto, la relación entre la expresión griega II como lectura OG y la expresión hebrea B parece segura, lo cual apunta a que el texto de la posible fuente hebrea de OG en 1 Re 15:33 tuviera tal expresión.

\subsubsection{La expresión griega IV}

En segundo lugar, hay otros seis sincronismos en 1 y 2 Reyes que

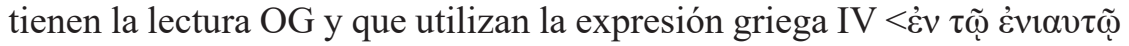

${ }^{23}$ Proponemos como posible lectura OG en este caso la que presentan la mayoría de los testimonios de LXX ya que la lectura del Códice Vaticano (B) es exclusiva de su testimonio, por lo tanto tiene toda la impresión de ser una lectura individual, y la que presentan los manuscritos antioquenos parece ser una lectura particular de su tradición, lo cual analizaremos en profundidad cuando veamos la expresión griega III.

24 SHENKel, CRD, págs. 49-50.

25 A saber, en: Gen 47:18, Lev 19:24, Lev 19:25, Lev 25:4, Lev 25:20, Lev 25:21, Num 9:1, Deut 26:12, 2 Cr 23:1, Ezr 3:8, Jer 25:1, Jer 26:2, Jer 28:59, Jer 43:9, Jer 46:1, Jer 52:4, Ez 20:1, Ez 24:1y Ez 29:1. Únicamente en 2 Cr 3:2 y en Dan 2:1 aparece la expresión II en griego con la A en hebreo. 
$\tau \tilde{\omega}$ [año]>. Estos sincronismos están en $1 \operatorname{Re} 14: 25,1 \operatorname{Re} 15: 9,1 \operatorname{Re}$ 16:28a, $1 \operatorname{Re} 18: 1 ; 1 \operatorname{Re} 22: 2$ y $1 \operatorname{Re} 22: 52$; en este último, la lectura OG únicamente está presente en los manuscritos antioquenos. De estos seis casos hay un hebreo igual detrás en cuatro de ellos.

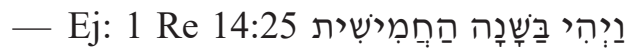

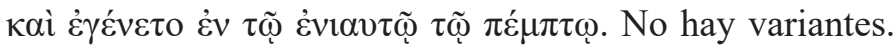

En los otros dos casos (1 Re 16:28a y 1 Re 22:52) no hay ningún texto hebreo detrás del griego para comparar, ya que su contenido sólo aparece en ciertos manuscritos de la tradición de LXX: el primero se omite en el Códice Alejandrino, en la versión Armenia y en la Sirohexapla ${ }^{26}$ y el segundo sólo aparece de esa manera en los manuscritos antioquenos boc $_{2} \mathrm{e}_{2}$.

- 1 Re 16:28a om A\&g

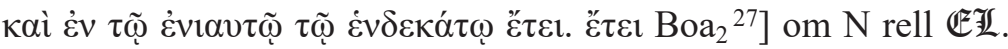

${ }^{26}$ En la historia del texto de la Septuaginta sobresalen principalmente dos procesos por los cuáles no se conservan lecturas OG en los manuscritos de LXX. Uno de ellos es el de la ya citada revisión kaige, por la cual el contenido de los manuscritos de LXX se cambió para reflejar mejor el griego del proto-TM, y el segundo tiene que ver con los cambios que se dieron en el contenido de la Antigua Septuaginta tras la elaboración de la Hexapla. Esta fue la obra coordinada y producida por Orígenes en Cesárea de Palestina entre los años 235 y 250 d. C. Consistía en una Biblia en seis columnas sinópticas, en las cuáles -por orden- estaba el texto hebreo masorético, ese texto transliterado al griego, la traducción de Áquila, la de Símaco, la Septuaginta y la de Teodoción. Desgraciadamente, la obra se perdió. No obstante, la columna de la Septuaginta se conservó en gran medida en la traducción al sirio de Pablo de Tella del siglo viI d. C. conocida por el nombre de Siro-Hexapla. En ella, además de en otros testimonios, se aprecian los citados cambios producidos en el texto de la Antigua Septuaginta, los cuáles se dieron porque Orígenes editó por separado su columna, la quinta, corrigiéndola según el texto hebreo en curso; TetLEY, Reconstructed, pág. 25. A causa de esto, testimonios de LXX como la propia Sirohexapla, el Códice Alejandrino y la versión Armenia no tienen, por ejemplo, la fórmula aquí citada de Josafat de 1 Re 16:28a, que el TM tampoco presenta.

27 Proponemos como lectura OG la que presentan la mayoría de los manuscritos de

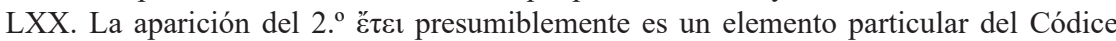
Vaticano ya que aparece únicamente en su testimonio y en dos manuscritos muy próximos a él, el a $\mathrm{a}_{2}$ y el antioqueno o; Aejmelaeus, «Kaige Readings», pág. 171. 


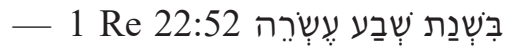

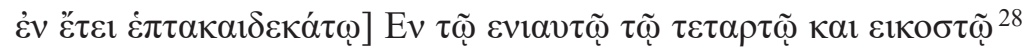
$\operatorname{boc}_{2} \mathrm{e}_{2}$

En los casos en los que hay hebreo detrás en todos ellos, salvo en 1

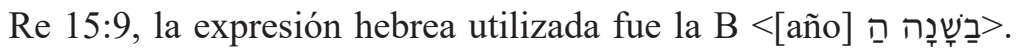

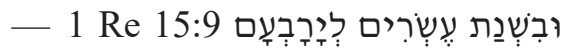

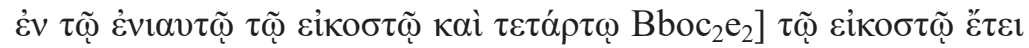

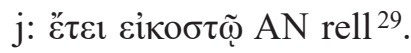

Si miramos el resto de textos de la Biblia Hebrea, se ve que la expresión griega IV aparece en seis sincronismos ${ }^{30}$, y en cuatro de ellos -esto es, salvo los dos del libro de Daniel en que siempre aparece la

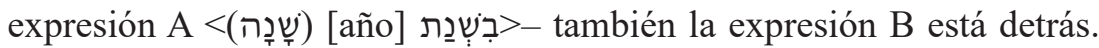

Por lo tanto, parece lógico que concluyamos que la expresión griega IV, tal y como expresó Shenkel, es una expresión OG que, junto con la II, lleva por debajo la expresión hebrea B. No obstante, aparece un problema con los textos de 1 Re 15:9; 1 Re 16:28a y 1 Re 22:52 que tienen sincronismos con números mayores de diez.

Tal y como hemos dicho previamente, no sólo en 1 y 2 Reyes sino también en la Biblia Hebrea, la expresión B no aparece con números mayores de diez. Además, como ya hemos mencionado, en los Óstraca de Samaría aparecen varios casos en los que encontramos la fórmula B en hebreo con números mayores de diez, confirmando la existencia de esta expresión con estos números. Es posible entonces que el traductor griego

${ }^{28}$ La lectura OG es la de los manuscritos antioquenos $\mathrm{boc}_{2} \mathrm{e}_{2}$, ya que el resto de los testimonios que presentan esta fórmula, como el Códice Vaticano, han visto afectado su contenido por la revisión kaige. Shenkel, CRD, págs. 64-68; Tetley, Reconstructed, pág. 132, y Hughes, Secrets, pág. 130. Por ello presentan una expresión y una cronología distinta a la de los manuscritos antioquenos.

${ }^{29}$ Hemos propuesto como lectura OG la del Códice Vaticano y los manuscritos antioquenos porque $\mathrm{j}$ tiene una lectura particular y porque el resto de los testimonios de LXX como el Códice Alejandrino tienen una lectura filo-TM de carácter hexaplar por la cual tienen una expresión y una cronología en el sincronismo igual que el TM y distinta del OG.

${ }^{30}$ A saber, en: Num 10:11, Jer 32:1, Jer 36:1, Jer 45:1, Dan 10:1 y Dan 11:1. 
tradujera de algún texto hebreo en el que se emplease esta fórmula con números mayores de diez - escritos probablemente en hierático- y que esto le llevase a traducir los sincronismos con la expresión IV y no con la II.

Finalmente, tenemos que responder a la pregunta de por qué una misma expresión hebrea pudo tener dos traducciones en griego. Entendemos que una de las posibilidades pudo ser que la expresión II fuera la forma mayoritaria para traducir la expresión hebrea $\mathrm{B}$, y que la IV fuera también traducción de B por la tendencia -observable no sólo en 1 y 2 Reyes $^{31}$ sino también en el resto de la Biblia Hebrea ${ }^{32}$ - a traducir el esquema compuesto por la preposición ב más la palabra correspon-

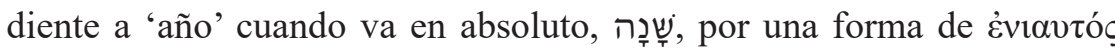

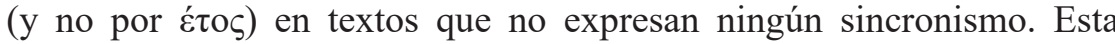
tendencia sería la causante de que hoy en nuestros textos tengamos no sólo la expresión griega II para traducir la $\mathrm{B}$ del hebreo sino también la IV. Se tendrá que seguir trabajando en el futuro para ver si aparecen más elementos que refuercen esta hipótesis o que, por el contrario, puedan apuntar en otra dirección.

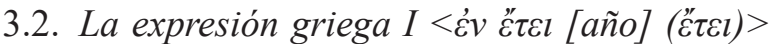

La expresión griega I aparece como OG en los sincronismos de las fórmulas de reinado de 1 Re 15:25 en 1 Reyes, en todos los sincronismos de las fórmulas de reinado de 2 Reyes y en sincronismos de fuera de las fórmulas de reinado como en 2 Re 18:10. En todos ellos la expresión hebrea

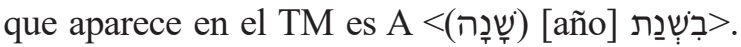

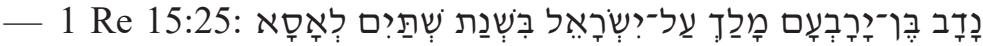

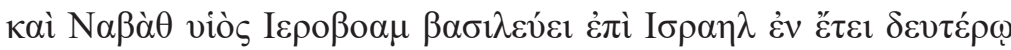
$\tau$ ○õ $A \sigma \alpha]$ No hay variantes en la estructura de la fórmula.

31 A saber, en: $1 \operatorname{Re} 4: 7$ y $1 \operatorname{Re} 5: 25=3 \operatorname{Reg} 5: 11$ y $1 \operatorname{Re} 9: 25=3 \operatorname{Reg} 2: 35 \mathrm{~g}$ y 1 $\operatorname{Re} 10: 14 ; 1 \operatorname{Re} 10: 25=3 \operatorname{Reg} 10: 28$ y $2 \operatorname{Re} 17: 4$.

${ }^{32}$ A saber, en: Gen 17:21, Gen 26:12, Gen 47:17, Ex 23:14, Ex23:17 y Ex 23:29, Ex 30:10, Ex 34:23, Lev 23:41, Lev 25:53, Deut 14:28, Deut 15:20, Deut 16:16, Jos 5:12, Jue 10:8, Jue 11:40, 1 Sam 1:7, 1 Sam 7:16, 2 Cr 8:13, 2 Cr 9:13 y 24, 2 Cr 24:5, Neh 10:33 y 35 y Zac 14:16. 


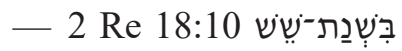

$\dot{\varepsilon} \nu$ हैं $\varepsilon 1$ ह̌ $\kappa \tau \omega]$ No hay variantes.

Además, si echamos un vistazo al resto de la Biblia Hebrea, vemos que la expresión griega I aparece $15^{33}$ veces cuando hay texto hebreo detrás y en todas ellas A está en el texto hebreo. Por tanto, creemos que no se puede concluir otra cosa que establecer una relación entre la expresión griega I y la expresión hebrea A.

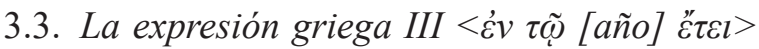

3.3.1. ¿Con qué se identifica la expresión III del griego?

En los libros de 1 y 2 Reyes tenemos doce ${ }^{34}$ textos en los que la expresión III en griego es claramente OG y cuatro ${ }^{35}$ en los que dudamos acerca de su carácter. De esos doce textos cinco ${ }^{36}$ están en fórmulas de reinado -las cuáles hemos visto que han sufrido cambios en varias ocasiones en el TM a A- uno no tiene texto hebreo detrás ${ }^{37}$ y siete están fuera de las fórmulas de reinado. De los siete que están fuera de las fórmulas de reinado, en cinco de ellos la expresión hebrea que está por

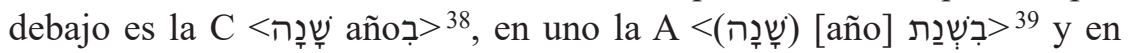
otro la B < [año]

La posición que sugerimos en este trabajo es la que plantea hacer una identificación plena entre los textos OG que tienen la expresión griega III

${ }^{33}$ A saber, en: 2 Cr 16:12, 2 Cr 17:7, Ezr 1:1, Ezr 5:13, Ezr 6:3, Ezr 7:7, Neh 13:6, Est 3:7, Dan 1:1, Dan 7:1, Dan 8:1, Dan 9:1, Dan 9:2, Dan 10:1 y Dan 11:1.

${ }^{34}$ A saber, en: 1 Re 6:1a, 1 Re 15:1, 1 Re 15:8, 1 Re 16:6, 1Re 16:23, 2 Re 12:7, $2 \operatorname{Re} 17: 6,2 \operatorname{Re} 18: 13,2 \operatorname{Re} 22: 3,2 \operatorname{Re} 23: 23,2 \operatorname{Re} 24: 12$ y $2 \operatorname{Re} 25: 27$.

35 A saber, en: $2 \operatorname{Re} 13: 1,2 \operatorname{Re} 14: 23,2 \operatorname{Re} 15: 1$ y $2 \operatorname{Re} 15: 8$.

36 A saber, en: $1 \operatorname{Re} 15: 1,1 \operatorname{Re} 15: 8,1 \operatorname{Re} 16: 6,1 \operatorname{Re} 16: 15$ y $1 \operatorname{Re} 16: 23$.

37 En 1 Re 15:8.

38 A saber, en: 1 Re 6:1a, $2 \operatorname{Re} 18: 13,2 \operatorname{Re} 22: 3,2 \operatorname{Re} 23: 23$ y $2 \operatorname{Re}$ 25:27.

39 En 2 Re 24:12.

40 En 2 Re 17:6. 
con la expresión hebrea $\mathrm{C}$, salvo en los dos últimos casos en los que aparece A y B. ¿En qué se justifica esta afirmación?

Además de las coincidencias de 1 y 2 Reyes entre la expresión griega III y la hebrea $\mathrm{C}$, si miramos el resto de la Biblia Hebrea vemos que la expresión griega III aparece treinta y seis veces con texto hebreo detrás ${ }^{41}$, de las cuáles en dieciocho ocasiones la fórmula hebrea que está detrás es $\mathrm{C}^{42}$-todas las ocasiones en las que $\mathrm{C}$ aparece fuera de $1 \mathrm{y}$ 2 Reyes-, en diez ocasiones $\mathrm{A}^{43}$, en cuatro $\mathrm{B}^{44}$ y en tres de forma anómala ${ }^{45}$. En las dieciocho ocasiones que la expresión griega III traduce $\mathrm{C}$ el número utilizado en el sincronismo es mayor de diez mientras que en las ocasiones en las que traduce A y B, únicamente en Gen 7:11 -texto que analizaremos posteriormente- y en $2 \mathrm{Cr}$ 34:8 el número utilizado en el sincronismo es mayor de diez. En el resto de los casos que III traduce A y B los números utilizados son igual o menores a diez.

Volviendo a 1 y 2 Reyes, los números de los sincronismos de 2 Re 24:12 y de $2 \operatorname{Re} 17: 6$ son igual o menores a diez, mientras que en el resto de casos son mayores de diez; algo que fuera de 1 y 2 Reyes y también en cinco de los siete sincronismos de fuera de las fórmulas de

41 A saber, en: Gen 7:11, Gen 8:13, Gen 14:4, Gen 14:5, Num 33:38, Deut 1:3, 1 Cr 26:31, 2 Cr 12:12, 2 Cr 13:1, 2 Cr 16:1, 2 Cr 27:5, 2 Cr 34:3a, 2 Cr 34:3b, 2 Cr 34:8, 2 Cr 35:19, Est 1:3, Est 2:6, Hag 1:1, Hag 1:15, Zac 1:7, Zac 7:1, Jer 25:3, Jer 35:1, Jer 46:2, Jer 52:31, Ez 1:1, Ez 8:1, Ez 26:1, Ez 29:17, Ez 30:30, Ez 31:1, Ez 32:2, Ez 32:17, Ez 33:21, Ez 40:1a y Ez 40:1b.

42 A saber, en: Gen 8:13, Gen 14:5, Deut 1:3, 2 Cr 34:3b, 2 Cr 35:19, Jer 25:3, Jer 46:2, Jer 52:31, Ez 1:1, Ez 26:1, Ez 29:17, Ez 30:20, Ez 31:1, Ez 32:1, Ez 32:17, Ez 33:21, Ez 40:1a y Ez 40:1b.

43 A saber, en: Gen 7:11, 2 Cr 13:1, 2 Cr 16:1, 2 Cr 34:8, Est 1:3, Est 2:6, Hag 1:1, Hag 1:15, Zac 1:7 y Zac 7:1

44 A saber, en: 2 Cr 12:2, 2 Cr 27:5, Jer 35:1 y Ez 8:1.

45 A saber, en: Num 33:38, 1 Cr 26:31 y 2 Cr 34:3. En los dos primeros tenemos el particular caso del número 40. Este aparece como ordinal en la Biblia Hebrea en tres

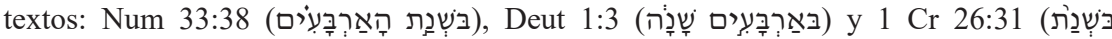

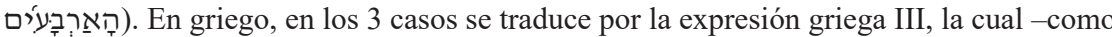
hemos visto- traduce la expresión hebrea C. Por ello, consideramos que los textos de Num 33:38 y 1 Cr 26:31 en hebreo han sufrido algún tipo de corrupción en su intento por adaptar la expresión hebrea $\mathrm{C}$ a la $\mathrm{A}$, y por eso en ellos el número 40 aparece determinado; algo anómalo y que no se observa en la Biblia Hebrea para números mayores de diez. 
reinado, se relaciona con la expresión hebrea C. Por todo ello, nos parece razonable sugerir que los sincronismos de 1 y 2 Reyes en los que se utilice la expresión griega III y el número que aparezca sea mayor que diez, el texto hebreo que estaría detrás es un texto con la expresión hebrea C. Las fórmulas de reinado de $1 \operatorname{Re} 15: 1,1 \operatorname{Re} 16: 6^{46}$, y $1 \operatorname{Re}$ 16:23 en la fuente hebrea de OG no tendrían la expresión A del TM sino la C. Del mismo modo, 1 Re 15:8, texto que no aparece en el hebreo masorético.

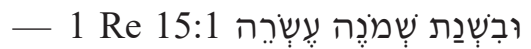

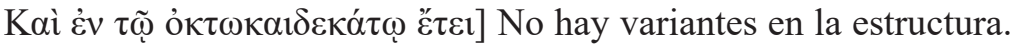

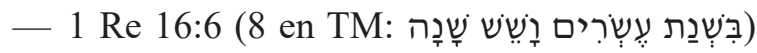

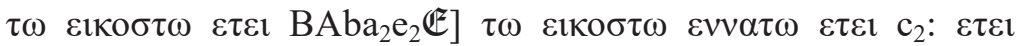

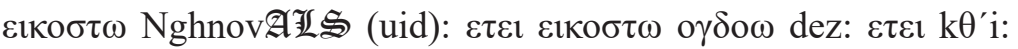

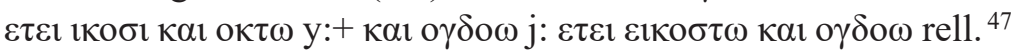

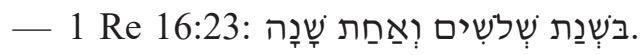

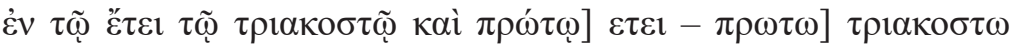

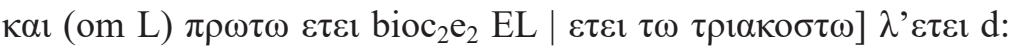

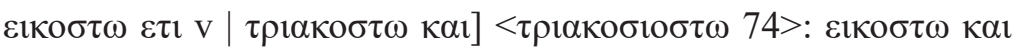
$\mathrm{N}^{*} \mathrm{xy}$ : om $\kappa \alpha$ Na?p | om $\kappa \alpha \imath \pi \rho \omega \tau \omega$ Jos | $\left.\pi \rho \omega \tau \omega\right] \varepsilon \beta \delta о \mu \omega$ $\mathrm{N}^{*}$ vxy:+ $\varepsilon \tau \varepsilon 1$ AS $(\text { sub } \div)^{48}$.

461 Re 16:8 en TM, por el desdoblamiento de la fórmula en LXX.

47 Proponemos como lectura OG el testimonio del Códice Vaticano, Alejandrino, los manuscritos antioquenos y la version Etiópica en conformidad con SHENKel, CRD, pág. 36. Pensamos que los otros manuscritos presentan en griego la expresión I, traducción de la A que presenta el TM en 16:8, debido a una contaminación hexaplar.

${ }^{48}$ La lectura OG que hemos propuesto en este trabajo es la de los manuscritos

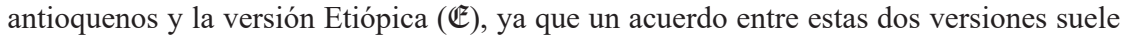
apuntar con seguridad a una lectura muy antigua y presumiblemente OG; Siegfried Kreuzer «B or not B? The Place of Codex Vaticanus in Textual History and in Septuagint Research», en Text-Critical and Hermeneutical Studies in the Septuagint (VT. S $\left.{ }_{157}\right)$, eds. J. S. Cook y J. Hermann (Leiden-Boston: Brill, 2012) págs. 69-96: 75. Por otra parte, el hecho de que el Códice Vaticano presente otra expresión parece explicarse como causa de una lectura particular, ya que frecuentemente su contenido coincide con el de la versión Etiópica, descartando por tanto que sea una lectura antigua; Aejmelaeus, «Kaige Readings», pág. 171. Finalmente, los testimonios $\mathcal{A g}$ presentan un segundo $\varepsilon \tau \varepsilon \iota$ reflejando la influencia de la Hexapla en su texto ya que el TM también tiene un segundo שָ̇̇ie Este caso es también un ejemplo de como los signos hexaplares fueron mal trans- 
$-1 \operatorname{Re} 15: 8$

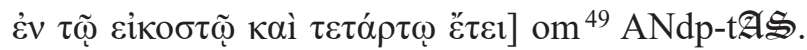

Por último, ¿qué sucede en los textos de 2 Re 24:12 -con expresión hebrea A-y en 2 Re 17:6 -con expresión hebrea B-, en los cuales aparece presumiblemente ${ }^{50}$ la expresión III como lectura OG, con un número en el sincronismo menor que diez? Para resolver esta cuestión tenemos que observar los casos fuera de 1 y 2 Reyes en dónde esté la expresión III como lectura OG con números menores que 10. En estos casos -todos ellos de textos que o bien forman parte del grupo de libros del Late Biblical Hebrew ${ }^{51}$ o bien se encuentran en libros cuyo hebreo se ha visto adaptado al de los libros de aquel grupo-, la expresión III es

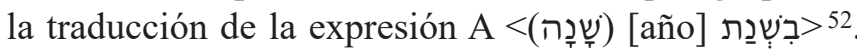

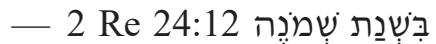

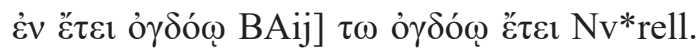

- $2 \operatorname{Re} 17: 6$

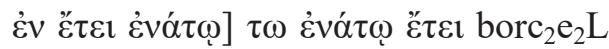

¿Por qué pudo pasar esto? Una posible respuesta la puede dar el texto de $2 \operatorname{Re} 25: 1$. Este texto con expresión $\mathrm{B}<$ año]

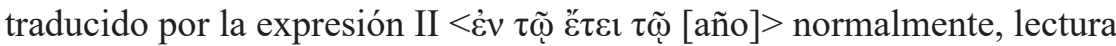
OG de la mayoría de manuscritos en este texto. Esto sucede también en

mitidos a lo largo de la transmisión del texto ya que aparece un óbelo - utilizado para indicar la presencia de material original de la Septuaginta en el TM - antes del segundo $\varepsilon \tau \varepsilon 1$ cuando lo que tendría que aparecer es un asterisco - utilizado para indicar la presencia de material agregado a la columna de la Septuaginta; PIQUER, «Minuses», pág. 63.

49 Omisión de carácter hexaplar por la cual el texto de tales manuscritos es igual que el del TM, que tampoco presenta el sincronismo.

50 Presumiblemente porque, como se ve en la nota 21, estamos en una sección kaige y en ella el OG es muy difícil de recuperar. Para ello se cuenta con los manuscritos antioquenos y las versiones secundarias, cuyo uso no está exento de problemas. Una exposición de los mismos se puede ver en la reciente tesis de Timo Texoniemi, A Game of Thrones: Textual History of 2 Kings 17 in Light of the Old Latin (Helsinki: University of Helsinki, 2019) págs. 14-15.

51 FASSBERG, «Late», págs. 1-15.

52 A saber, en: Gen 7:11, 2 Cr 13:1, 2 Cr 16:1, 2 Cr 34:8, Est 1:3, Est 2:6, Hag 1:1, Hag 1:15, Zac 1:7 y Zac 7:1. 
el texto de 1 Re 15:33, en donde se ha señalado que L tiene una lectura particular de la expresión III y B una lectura OG con la expresión II que debajo tendría la B. ¿Por qué L tiene la expresión III $<\dot{\varepsilon} \vee \tau \tilde{\omega}$ [año] हैं $\tau \varepsilon>$ ?

No pocos autores han afirmado que los manuscritos antioquenos tienen como uno de sus rasgos el empleo de aticismos, es decir, de mejorar el griego de las traducciones de acuerdo al griego clásico ${ }^{53}$. Quizá a esto obedezca la aparición de III en los sincronismos con números iguales o menores que diez en los que $\mathrm{C}$ ב ב no es su Vorlage en la Biblia,

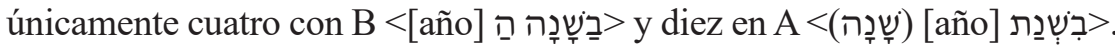
El uso de la expresión III en estos casos puede entenderse como un intento de escribir la traducción del hebreo en un buen griego, según el cual en la expresión del sincronismo el adjetivo va antes que el nombre ${ }^{54}$.

\subsubsection{Una fórmula a caballo entre la $\mathrm{C}$ y la $\mathrm{A}$ del hebreo}

Sobre $1 \operatorname{Re} 15: 1 ; 1 \operatorname{Re} 15: 8 ; 1 \operatorname{Re} 16: 8 ; 1 \operatorname{Re} 16: 15$ y $1 \operatorname{Re} 16: 23$, cabe destacar un elemento que comparten las tres últimas de las cuatro fórmulas que aparecen en el TM y que, posiblemente, es una de las aportaciones más novedosas de este trabajo, el שָָָ adicional ${ }^{55}$. El único que ha tratado de nuevo este שָָָ ha sido Shenkel, según el cual

The Syrohexapla translates this שִָָ more frequently (2 Re 13:1, 10; $14: 23 ; 15: 1,8$ ), attributing the addition to Aquila and the Quinta at 14:23, but no version seems to have translated the addition consistently ${ }^{56}$.

No compartimos enteramente esta concepción sobre la adición de la palabra para 'año' en hebreo y apostamos porque se trata de una eviden-

53 Fernández Marcos, Septuaginta, pág. 194.

${ }^{54}$ Emilio Crespo, Luz Conti y Helena Maquieira, Sintaxis del griego clásico (Madrid: Gredos, 2003).

55 Son las fórmulas de 1 y 2 Reyes en las que hemos visto al principio del punto 2.3.1 que tienen la expresión III como lectura OG. El único de los cinco textos en los que ese ש்ָָׁ adicional no aparece en el TM es el de 1 Re 15:1 ya que, como se indica en la nota 32, la fórmula de 1 Re 15:8 no aparece en el TM.

56 SHENkel, CRD, pág. 49. 
cia de la adaptación que se hizo en el TM de Reyes y que también se encuentra en otros textos de la Biblia como Gen 7:11 ${ }^{57}$ para adecuar la expresión hebrea $\mathrm{C}$ a la expresión hebrea $\mathrm{A}$. ¿Qué pruebas hay de esto? Si observamos por ejemplo los textos de 1 Reyes que suponemos que tienen la expresión $C$ detrás, en todos ellos ${ }^{58}$ exceptuando 1 Re 15:1, este שָָָׁ se repite, en todos el número del sincronismo es mayor que

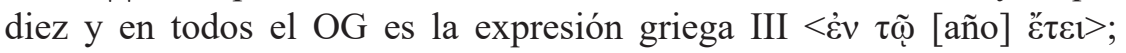
expresión que para números mayores que diez se identifica con C. Fuera de Reyes, el texto de Gen 7:11 es la única prueba de la expresión hebrea A con números mayores de diez. La expresión hebrea A hemos comprobado supra en el apdo. 2.2. que pertenece al grupo del Late Biblical Hebrew. ¿Por qué aparece en el Pentateuco? Viendo el texto de Gen 7:11 se comprueba que tiene ese שָָׁ adicional, que el número del sincronismo es mayor de diez y que los textos griegos tienen la expresión III; por lo tanto, la expresión que probablemente tendría este texto en origen sería la $\mathrm{C}$.

En definitiva, parece que es perfectamente lógico plantear que de una fórmula tipo $\mathrm{C}$ >

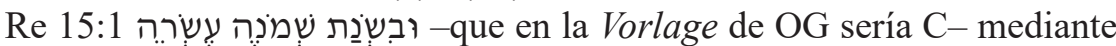

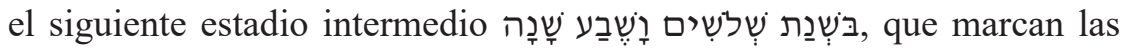
anteriores fórmulas. No obstante, ¿son los textos que tienen la expresión griega III como OG los únicos en los que en el TM aparece esta fórmula בְשְׁנת [año medio camino entre C y A, o hay más? Para responder a esta pregunta, tenemos que resolver el tercer grupo de interrogantes planteado en la introducción a este trabajo.

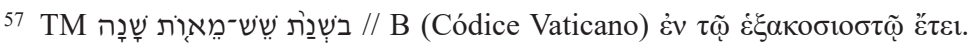

$581 \operatorname{Re} 16: 8$ : 16.

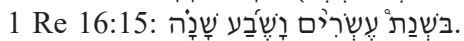

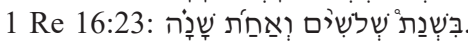

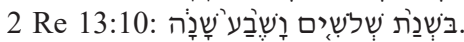


4. ¿Por Qué el Texto Masorético y el de la SUPUesta fuente HEBREA DE LXX UTILIZAN FORMAS DISTINTAS PARA EXPRESAR LOS SINCRONISMOS DE LAS FÓRMULAS DE REINADO DE 1 Y 2 REYES?

La respuesta a la cuestión de si hay más textos en el TM, junto con los anteriores que en su lectura OG tienen la expresión griega III, que

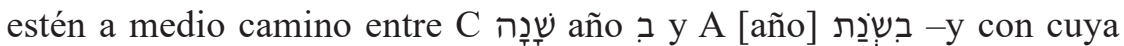
resolución se da respuesta a este tercer grupo de interrogantes- es positiva. Esta fórmula a medio camino entre C y A, בְשַַׁת [año, aparece en $1 \operatorname{Re} 16: 29^{59}$-texto discutido más abajo-; 2 Re 8:25 $5^{60} ; 2$ Re $13: 1^{61} ; 2 \operatorname{Re} 13: 10^{62} ; 2 \operatorname{Re} 14: 23^{63} ; 2 \operatorname{Re} 15: 1^{64} ; 2 \operatorname{Re} 15: 8^{65} ; 2 \operatorname{Re}$ 15:13 ${ }^{66} ; 2 \operatorname{Re} 15: 17^{67} ; 2 \operatorname{Re} 15: 23^{68} ; 2 \operatorname{Re} 15: 27^{69}$ y $2 \operatorname{Re} 16: 1^{70}$, en definitiva, en todos los sincronismos -salvo los que utilizan la expresión B < [año] 1 Re 22:52- de 1 y 2 Reyes, cuya fecha de sincronización incluye un número mayor que diez menos el ya analizado 1 Re 15:1 y 2 Re 3:1

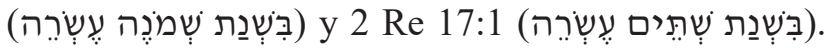

Con esto defendemos que en 1 y 2 Reyes se utilizaron primero dos formas para expresar los números mayores que diez en los sincronismos, la fórmula B para los casos de 1 Re 15:9, 16:28a y 22:52 y la fórmula $\mathrm{C}$ para el resto. ¿Por qué no se tiene ni la fórmula $\mathrm{B}$ ni la $\mathrm{C}$ completa

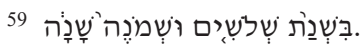

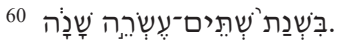

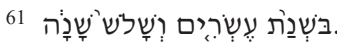

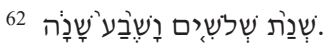

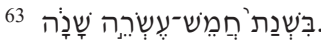

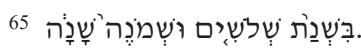

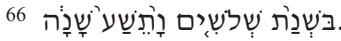

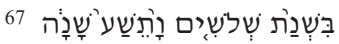

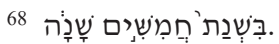

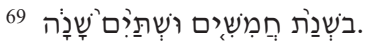

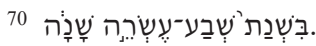

64 Único caso en el que el numeral no es compuesto pero que, pensamos, no presenta problemas ya que la característica común por la cual se usa la expresión $\mathrm{C}$ en estos textos es que el número que se expresa es mayor que diez: 
en ninguno de los otros casos en el TM? No es descabellado pensar que el hebreo de 1 y 2 Reyes sufrió en un momento anterior a la traducción de LXX de Reyes una revisión como la tipo kaige del griego (filomasorética) en la cual se armonizaron las formas de expresar ciertos elementos con el lenguaje de la época -expresión tipo A- y que afectó en principio más a 2 Reyes que a 1 Reyes, algo ya planteado por Julio Trebolle, Pablo Torijano y Andrés Piquer en diversas publicaciones ${ }^{71}$.

Prueba de ello son los cambios reseñados en la forma de expresar el sincronismo. Como hemos visto, en 1 Reyes el TM expresa todos los sincronismos con números mayores que diez con la expresión A. Sin embargo, el texto griego informa que en ciertos casos su fuente hebrea fue la B-1 Re 15:9, 1 Re 16:28a y 1 Re 22:52- y en otros la C - 1 Re 15:1, $1 \operatorname{Re} 15: 8,1 \operatorname{Re} 16: 6,1 \operatorname{Re} 16: 15$ y 1 Re 16:23-. El TM armonizó a todos con la expresión A dejando evidencias de ese proceso de armonización en textos como 1 Re 16:6, 15 y 23.

Por el contrario, en 2 Reyes ese proceso de armonización parece ser que afectó casi en igual medida a la Vorlage del OG y al hebreo masorético, ya que el traductor del OG empleó en estos casos la expresión

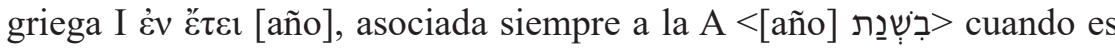
OG. Prueba de ello es que en las fórmulas de reinado de 2 Reyes no tenemos evidencia alguna de que el hebreo haya podido ser distinto al del hebreo masorético. De lo que tenemos tiene evidencia, tal y como hemos señalado antes, es que salvo en $2 \operatorname{Re} 3: 1$ y $2 \operatorname{Re} 17: 1$ en todas las demás fórmulas que utilizan números mayores que diez hay restos

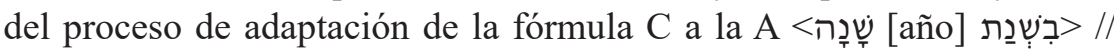

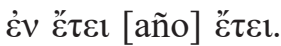

Finalmente, ¿qué pasa con las fórmulas de 1 y 2 Reyes -salvo 15:33cuando tienen expresado un sincronismo con un número menor o igual a diez pero utilizan la expresión hebrea A? Estas son las fórmulas de

71 Andrés Piquer y Pablo Toridano, «Between the Search in the Word and the Asking to God: Two Mantic Verbs in the Textual History of Samuel-Kings», en In the Footsteps of Sherlock Holmes, eds. Kristin De Troyer, Timothy M. Law y Marketta Kilkeström (Leuven: Peeters, 2014) págs. 299-330, y Julio Trebolle, Pablo ToriJano y Andrés Piquer, «Algunas características distintivas del texto masorético de 1 libro de Reyes: Desmitologización polémica y substitución de términos mánticos», Cadmo 15 (2005) págs. 9-21. 
$1 \operatorname{Re} 15: 25 ; 1 \operatorname{Re} 15: 28 ; 1 \operatorname{Re} 16: 29 ; 2$ Re 1:17; 2 Re 8:16-17; 2 Re 10:36; $2 \operatorname{Re} 12: 1 ; 2 \operatorname{Re} 14: 1 ; 2 \operatorname{Re} 15: 32$ y $2 \operatorname{Re} 18: 1$.

Desde nuestro punto de vista, las fórmulas de 2 Reyes en las cuáles A está presente en el TM y I en toda la Septuaginta pueden haber sufrido el mismo proceso de revisión filomasorética que las fórmulas de 2 Reyes con sincronismos mayores que diez. Aunque no se tenga evidencia textual, por lo que hemos visto es más lógico pensar que de una expresión

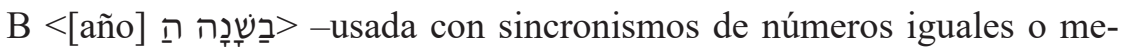

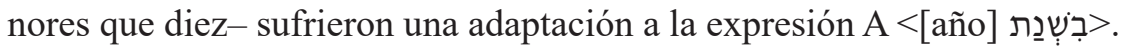
Por eso el OG, al igual que en los anteriores casos, tradujo con la expresión griega $\mathrm{I}<\dot{\varepsilon} v$ Ě $\varepsilon \varepsilon 1[$ año] $>$.

En segundo lugar, ¿qué pasa con los sincronismos de 1 Re 15:25 y 1 Re 16:29? El primer caso, cuyo sincronismo ya se ha analizado en el punto 2.2, se refiere a la fórmula de acceso de Nadab de Israel y el segundo a la de Ahab de Israel. Si se observa la estructura de la fórmula

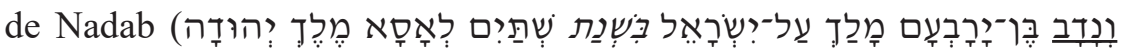

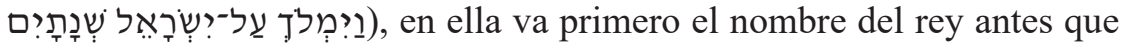
el sincronismo, algo anormal según la práctica totalidad de los autores ${ }^{72}$.

En el TM de 1 Reyes únicamente hay otros tres casos en los que el nombre del rey va primero antes que el sincronismo: 1 Re 22:41; $1 \operatorname{Re} 16: 29$ y $1 \operatorname{Re} 22: 52$. Estos tres casos no aparecen ni con el mismo contenido ni con la misma forma en el texto del OG, algo que hemos demostrado en este trabajo y ha sido señalado por otros autores ${ }^{73}$. Por lo tanto, ese orden en la fórmula denota arreglos en la cronología e inserciones tardías que tienen mayor presencia en el TM que en la Vorlage hebrea de OG. Teniendo en cuenta esto y que todos los textos de LXX presentan la fórmula de Nadab con la misma estructura y con los mismos datos que en el TM cabe hacerse la siguiente pregunta:¿la fórmula de Nadab era igual en la supuesta fuente hebrea del OG y por lo tanto los textos que se tienen sobre ella en griego pueden considerarse OG o pudo ser el hebreo del OG distinto que el del TM y no se conserva prueba de

72 Benjamin D. Thomas, Hezekiah and the Compositional History of the Book of Kings (Tübingen: Mohr Siebeck, 2014), y Jonathan MiLEs RoBkER, The Jehu Revolution: A Royal Tradition of the Northern Kingdom and its Ramifications (Berlin: De Gruyter, 2012).

73 Shenkel, CRD, pág. 48, y Tetley, Reconstructed, pág. 86. 
ello en griego porque todos los textos de LXX han sido afectados por recensiones o revisiones hebraizantes?

Desde nuestro punto de vista, el hebreo del OG en este caso pudo ser igual que el del TM como consecuencia de la misma revisión filomasorética que afectó casi en su práctica totalidad a 2 Reyes. Por tanto, el texto griego de Nadab se puede considerar OG. Prueba de ello es

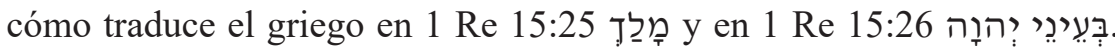

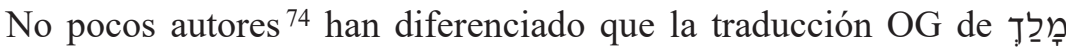
es la del presente histórico $\beta \alpha \sigma \imath \lambda \varepsilon v ́ \varepsilon ı$ a diferencia del aoristo $\dot{\varepsilon} \beta \alpha \sigma i ́ \lambda \varepsilon v \sigma \varepsilon v$

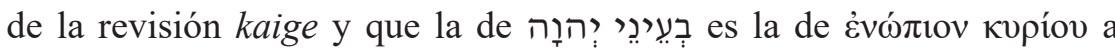

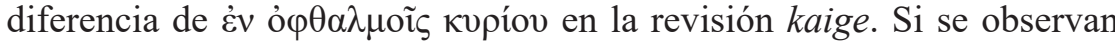
los textos kaige de 1 Re 22:41 y de $1 \operatorname{Re} 22: 52$ se ve que la primera

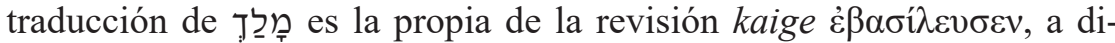
ferencia del presente histórico $\beta \alpha \sigma \imath \lambda \varepsilon v ́ \varepsilon ı$ propio del OG en 15:25. Del

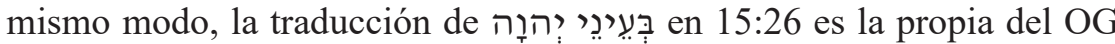

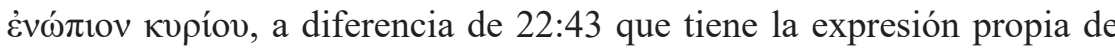

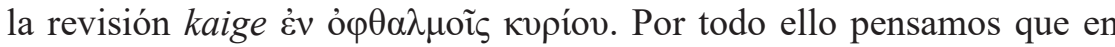
este caso es más probable que el cambio haya sido en el hebreo del OG y no una revisión hebraizante del griego.

En cuanto a la fórmula de Ahab, al igual que con la de Nadab, pensamos que no se puede dar una respuesta definitiva a esta cuestión ya que las dos opciones son plausibles. Sin embargo, del mismo modo que

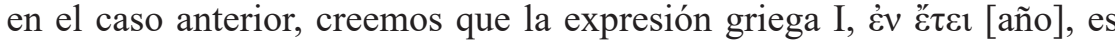
un texto OG y que su supuesta fuente hebrea tuvo la misma expresión que aparece en el TM para la formulación del sincronismo, la $\mathrm{A}<$ [año] בִּשְנַת> $>$.

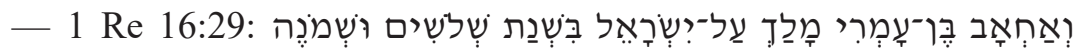

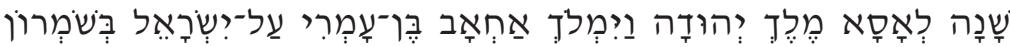

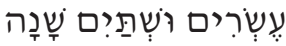

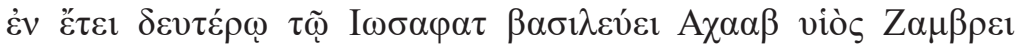

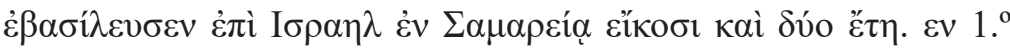

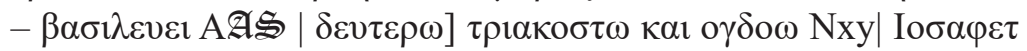

74 Shenkel, CRD, págs. 50-51; PessoA, Kaige 2 Samuel, pág. 26, y Albert.T. OlmSTEAD, «The Earliest Book of Kings», The American Journal of Semitic Languages and Literatures 31 (1915) págs. 169-214. 
$\left.\mathrm{E}: \alpha \sigma \alpha \mathrm{Nxy} \mid \beta \alpha \sigma 1 \lambda \varepsilon v \varepsilon 1 \mathrm{Bboc}_{2} \mathrm{e}_{2}\right] \varepsilon \beta \alpha \sigma 1 \lambda \varepsilon v \sigma \varepsilon v i$ : om $\mathrm{N}$ rell|

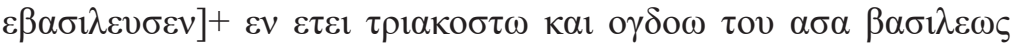
$10 v \delta \alpha \beta \alpha \sigma i \lambda \varepsilon v \sigma \alpha \varsigma \delta \varepsilon \alpha \chi \alpha \alpha \beta$ vios $\zeta \alpha \mu \beta \rho \imath \varepsilon \pi 11 \sigma \lambda$ AAS $[\varepsilon v-1 \sigma \lambda$ sub ※马]

Ello es así, principalmente por dos razones. La primera tiene que ver con el hecho de que la expresión griega I es la única expresión utilizada en todos los testimonios de LXX en esta fórmula, no existiendo ninguna variante de la misma, lo que reduce las posibilidades de su aparición a dos: o bien es un texto OG o, por el contrario, un texto filo-TM muy antiguo de LXX al estar presente en todos sus testimonios.

El motivo por el cual la balanza se inclina hacia la primera opción tiene que ver con la segunda de las razones. Esta es que en la fórmula de $1 \operatorname{Re} 16: 29$ se pueden apreciar perfectamente las diferencias entre los testimonios de LXX cuyo contenido ha sido adaptado al del TM como el Códice Alejandrino, la versión armenia y la Sirohexapla, y los que tienen elementos característicos del OG en esta sección como el Códice Vaticano y los manuscritos antioquenos. Los primeros presentan el orden de la fórmula del TM -nombre del rey antes del sincronismo- y la misma cronología -año treinta y ocho de Omrí-, mientras que los segundos tienen el orden de primero el sincronismo y luego el nombre del rey, la cronología del OG -año segundo de Josafat-, distinta en este punto de la del TM, y elementos característicos del OG como la presencia de verbos en presente histórico, $\beta \alpha \sigma i \lambda \varepsilon v \varepsilon^{75}{ }^{75}$.

Por todo ello es más lógico pensar que la presencia de la expresión I $\dot{\varepsilon} v$ É $\tau \varepsilon 1$ [año], en la fórmula de 1 Re 16:29 es una lectura OG que tiene que ver con que la supuesta fuente hebrea de debajo del OG tenía la misma expresión para formular el sincronismo que la que aparece en el TM, la A [año] בְשְַׁת debido a esa revisión filomasorética producida a nivel del hebreo antes de la traducción de la Septuaginta de 1 y 2 Reyes.

75 SHENkel, CRD, págs. 61-62. 


\section{CONClusiones}

En la introducción de este trabajo planteábamos que el propósito del mismo era ofrecer una respuesta a las siguientes cuestiones:

- ¿A qué se debe la aparición de distintas formas en hebreo para expresar los sincronismos? ¿Cuáles son las diferencias entre las expresiones A, B y C?

- ¿Por qué aparecen cuatro formas distintas en griego para traducir los sincronismos de dentro y fuera de las fórmulas de reinado del Libro de los Reyes? ¿Las diferencias entre ellas obedecen a cambios en la forma de traducir una misma expresión hebrea o tienen como base el uso de expresiones hebreas distintas?

- ¿Por qué el TM y el de la presunta fuente hebrea de LXX utilizan formas distintas para expresar los sincronismos de las fórmulas de reinado del Libro de los Reyes? ¿Por qué en las fórmulas de reinado del TM la variedad en las formas utilizadas para expresar los sincronismos no es la misma que fuera de las fórmulas de reinado?

Los resultados obtenidos en los distintos apartados de este trabajo nos permiten concluir que se ha podido dar una respuesta sólida a cada una de ellas. A saber:

Respecto de la primera cuestión, en cuanto a las diferencias entre las

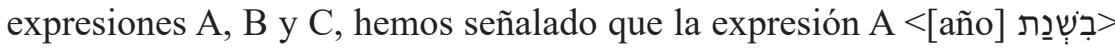
formula los sincronismos mediante una cadena constructa de dos sustantivos en la que el número - sin limitación por la cantidad expresada- va sin el artículo y en forma cardinal pero con significado ordinal. La ex-

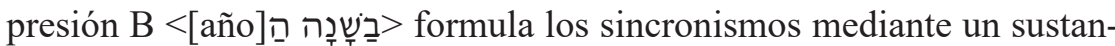
tivo, la palabra año, y un adjetivo, el numeral en forma ordinal, ambos determinados, con números del 1 al 10 en la práctica totalidad de textos de la Biblia y con números mayores de diez en los Óstraca de Samaría.

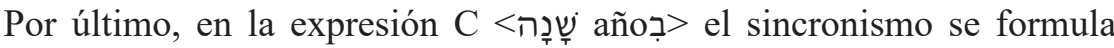
con el numeral en forma cardinal precedido por la preposición ב y seguido por la palabra año, únicamente utilizándose con numerales mayores que diez. 
En relación con cuál sea la razón de la aparición de estas tres formas en hebreo para expresar los sincronismos, la respuesta la hemos obtenido tras analizar en qué libros de la Biblia Hebrea aparecen cada una de ellas.

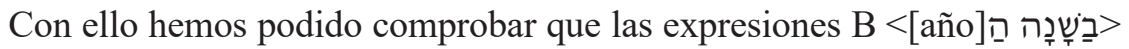

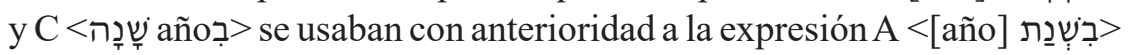
ya que las dos primeras se concentran en los libros que pertenecen al grupo del Classical Biblical Hebrew y la segunda en los que pertenecen al grupo del Late Biblical Hebrew. La expresión B se utilizaba con números del 1 al 10 y la expresión $\mathrm{C}$ con números mayores que 10. El hecho de que la expresión B aparezca en los Óstraca de Samaría del siglo viII a. C. y que en ellos también se utilice con números mayores que diez abre la puerta a la posibilidad de que quizá esta expresión, la más antigua atestiguada en el hebreo epigráfico, pudo preceder a la $\mathrm{C}$ y también se utilizó con números mayores que diez. La expresión A fue más tardía que estas expresiones y con ella se emplearon todo tipo de números, por ello su aparición en los libros del Late Biblical Hebrew coincide con la desaparición en los mismos de las expresiones B y C.

Respecto de la segunda cuestión, el análisis de cada uno de los anteriores apartados nos permite concluir que la razón por la cual aparecen cuatro expresiones distintas en griego para formular los sincronismos del Libro de los Reyes se basa en el hecho de que cada una de ellas tenía un texto hebreo distinto, aunque también se hayan dado cambios en la forma de traducir una misma expresión hebrea.

Hemos podido comprobar que las expresiones griegas II $<\dot{\varepsilon} v \tau \tilde{\varphi}$ हैं $\tau \varepsilon 1$

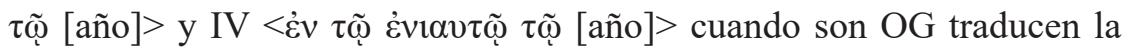

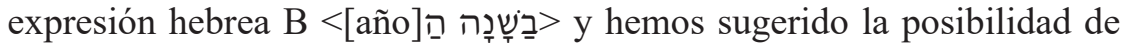
que quizá el hecho de que aparezcan en los sincronismos de Reyes dos expresiones para traducir la expresión hebrea B se deba a que de estos sincronismos los que contienen números mayores que 10 hayan estado escritos en la Vorlage del OG con signos en hierático, tal y como están los de los Óstraca de Samaría, en los que los signos hieráticos se utilizan también para los sincronismos mayores que 10 . Hemos podido comprobar también que la expresión griega $\mathrm{I}<\dot{\varepsilon} v$ ह̌ं $\tau \varepsilon ı$ [año] $>$, cuando se trata de OG, traduce un texto hebreo en el que la expresión utilizada es la A $<$ [año] בִשְַַׁת > También hemos visto que esta expresión ha sido utilizada en los textos griegos que han sufrido revisiones hebraizantes para 
adaptar su contenido al TM, como así reflejan textos que han sufrido estas adaptaciones como la hexaplar en el códice Alejandrino en la fórmula de 1 Re 16:15. Por otra parte, hemos comprobado que cuando la expresión griega III $<\dot{\varepsilon} v \tau \tilde{\varphi}$ [año] हैं $\varepsilon>>$ se utiliza con números mayores que 10 y es $\mathrm{OG}$, el texto hebreo del que traduce tendría la expresión

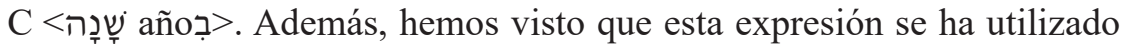
con números igual o menores a 10 como un intento de escribir la traducción del hebreo en un buen griego.

Respecto de la última de las cuestiones, hemos demostrado que en la presunta Vorlage hebrea de 1 y 2 Reyes se utilizaron primero dos formas para expresar los números mayores que diez en los sincronismos, la

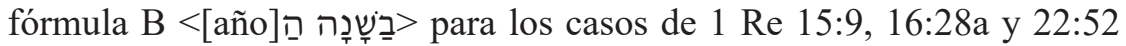

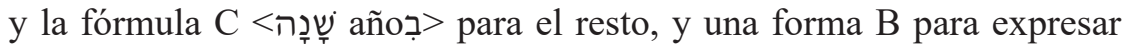
los sincronismos menores de 10 como el de 1 Re 15:33. También, que el hebreo de Reyes pudo haber sufrido en el período persa o helenístico una recensión como la tipo kaige del griego en la cual se armonizaron las formas de expresar ciertos elementos con el lenguaje de la época -expresión tipo A- y que afectó en principio más a 2 Reyes que a 1 Reyes. Por ello, en las fórmulas de reinado de 2 Reyes siempre se utilizó la expresión hebrea A. Como consecuencia de esto, el traductor

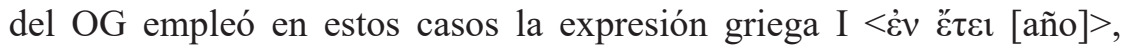
asociada siempre a la fórmula $\mathrm{A}<$ [año]

Por el contrario, en 1 Reyes, el TM expresa todos los sincronismos con la expresión A. Ello se debe a que completó ese proceso de revisión a la hebrea que afectó primero a 2 Reyes y armonizó todos los sincronismos de las fórmulas de reinado con la expresión que en la Vorlage hebrea también estaba presente en ese libro en la práctica totalidad de los sincronismos, la A. Una evidencia en el TM de ese proceso de adaptación de las expresiones $\mathrm{B}$ y $\mathrm{C}$ la $\mathrm{A}$ es la fórmula que hemos identificado

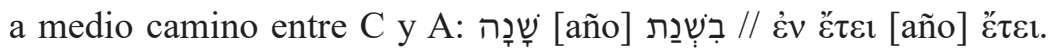

No compartimos enteramente la concepción de Shenkel sobre la adición de la palabra para 'año' en hebreo, en lo que él identificó como una variante inconsistente de la expresión A y hemos demostrado que es una evidencia de la adaptación que se hizo en el TM de Reyes para adecuar la expresión hebrea $\mathrm{C}$ a la expresión hebrea $\mathrm{A}$. ¿Qué pruebas hemos ofrecido de esto? Hemos visto que en los textos en los que dicho 
שָָׁ se repite, 1 Re 16:23, que en todos ellos los números son mayores que diez y en su práctica totalidad la expresión griega empleada es la III $<\dot{\varepsilon} \vee \tau \tilde{\omega}$ [año] हैं $\tau \varepsilon>$, algo identificado con la expresión C.

Por último, respecto de los sincronismos de 1 Re 15:25 y 1 Re 16:29, el primero ha servido de ejemplo para ilustrar que esa revisión kaige a la hebrea también afectó puntualmente a 1 Reyes, ya que el hebreo del OG fue igual aquí que en el TM.

Finalmente, concluimos este trabajo destacando las cuestiones que deben ser investigadas con mayor hincapié en futuras investigaciones, ya que la explicación que aquí hemos dado sobre las mismas no ha cerrado definitivamente todos los interrogantes que las rodean.

La primera de ellas es el hecho de que la expresión B parece que en el OG se haya traducido de dos formas distintas, con las expresiones griegas II y IV. Será necesario profundizar en la hipótesis sobre la relación que pudo tener la expresión griega IV con la posible existencia en la expresión $\mathrm{B}$ hebrea de números escritos en hierático.

La segunda de ellas tiene que ver con el hecho de que la expresión III del griego haya aparecido en algunos textos como lectura OG en sincronismos con números menores de 10. Estos textos han aparecido en los manuscritos antioquenos principalmente y en 2 Reyes, 2 Re 24:12 y 2 Re 17:6. Por ello, siendo conscientes de la complejidad de este libro para determinar lecturas OG, de la aparición de estos sincronismos en los manuscritos antioquenos y del hecho de que su lectura se pueda asociar a aticismos, habrá que determinar en el futuro si en verdad estos textos fijados como OG de 2 Reyes tienen en realidad una lectura OG o una propia de los manuscritos antioquenos.

En tercer lugar, se tendrá que seguir profundizando en la revisión filomasorética producida a nivel del hebreo antes de la traducción de la Septuaginta de 1 y 2 Reyes. Elementos de tal revisión fueron primeramente identificados, como hemos mencionado anteriormente, por Piquer, Torijano y Trebolle. En este trabajo hemos aportado nuevos elementos que presumiblemente formarían parte de esa revisión, como la adaptación en la formulación de los sincronismos a la expresión hebrea $\mathrm{A}$. Se tendrá que seguir investigando en torno a esta revisión para tratar de descubrir más características que se asocien a ella y poder así intentar delimitar las circunstancias de su elaboración. 
Por último, también será preciso abordar si el uso de la expresión

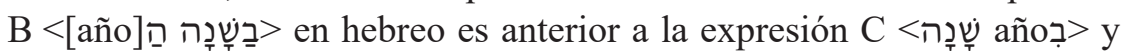
las diferencias que haya podido haber, si las hubo, en el empleo de estas expresiones entre los reinos de Israel y Judá. Todo ello ayudaría enormemente a realizar una aproximación a la difícil cuestión de la cronología de los reyes de Israel y Judá.

Recibido: 28/08/2019

Aceptado: 25/10/2019 